\title{
As contribuições da educação permanente em saúde no processo de trabalho da estratégia saúde da familia
}

\author{
The contributions of permanent health education to the \\ work process of the family health strategy
}

Guilherme Emanuel Weiss Pinheiro', Marcelo Schenk de Azambuja², Andrea Wander Bonamigo ${ }^{2}$

1 Universidade Federal de Pelotas, Pelotas (RS), Brasil.

${ }^{2}$ Universidade Federal de Ciências da Saúde de Porto Alegre, Porto Alegre (RS), Brasil.

\section{RESUMO}

Objetivo: Identificar as contribuições da educação permanente em saúde no processo de trabalho da estratégia saúde da família de um município do sul do Brasil. Método: Trata-se do recorte de um estudo de mestrado, com abordagem qualitativa, sendo utilizada a técnica da pesquisa-ação. Os participantes foram 33 profissionais de diferentes níveis de formação. A coleta de dados ocorreu a partir de um questionário autoaplicável sobre o processo educativo, os dados foram tratados por análise temática. Resultados: Surgiram três categorias de informações, como: contribuições da educação permanente no processo de trabalho junto aos usuários; contribuições das ações educativas no processo de trabalho com a equipe; e na relação com a gestão. Conclusão: A educação permanente está associada ao fazer cotidiano dos trabalhadores, auxiliando na construção de um cuidado centrado nos usuários, colaborando com a melhoria da qualidade dos serviços. Ainda, este estudo facilitou a implantação de uma Política Municipal de Educação Permanente em Saúde.

PALAVRAS-ChAVE: Educação continuada. Saúde da família. Atenção primária à saúde.

Recebido: Out. 27, 2017 Aceito: Maio 22, 2019

\section{COMO CITAR ESTE ARTIGO} Pinheiro GEW, Azambuja MS Bonamigo AW. As contribuições da educação permanente em saúde no processo de trabalho da estratégia saúde da família. Interdisciplinary Journal of Health Education. 2019 Jan-Dez;4(1-2):1-8. https://doi. org/10.4322/ijhe.2018.008

\section{CORRESPONDÊNCIA}

Guilherme Emanuel Weiss Pinheiro

Rua Brasília, 60, Centro, CEP: 95995-000,

Arvorezinha (RS), Brasil.

Tel.: (51) 98016-7020

enfermeiro.guipinheiro@gmail.com

\section{ABSTRACT}

Objective: Identify the contributions of permanent health education to the work process of the family health strategy in a city in South Brazil. Methods: The work is part of a MSc study and used a qualitative approach, with the technique of action research. A total of 33 professionals with different levels of training were included. Data were collected with a self-administered questionnaire about the educational process and were analyzed by thematic analysis. Results: Three categories of information: contributions of the permanent education in the process of working with users; contributions of the educational actions in the process of work with the team; and in relation to the management. Conclusion: Permanent education is associated to the daily life of workers, helping with the construction of care centered on users and contributing with the improvement of the quality of services. In addition, this study contributed with the implementation of a Municipal Policy of Permanent Health Education.

KEYWORDS: Education continuing. Family health. Primary health care.

\section{FONTE DE FINANCIAMENTO}

Nenhuma.

\section{CONFLITO DE INTERESSE}

Os autores declararam não

haver conflitos de interesse.

O estudo foi realizado na

Secretaria Municipal de Saúde

de Arvorezinha (RS), Brasil. 


\section{Introdução}

As práticas educativas no contexto do Sistema Único de Saúde (SUS) vêm ocorrendo de forma sistemática e contínua ao longo dos últimos anos, especialmente após a implementação da Política Nacional de Educação Permanente em Saúde (PNEPS), que traz conceitos importantes para a formulação de um processo educativo no trabalho. Assim, a Educação Permanente em Saúde (EPS), é entendida como educação no trabalho e tem como um dos seus principais objetivos qualificar e transformar as práticas de trabalho no SUS ${ }^{1}$. Segundo Pinto $^{2}$, a EPS é vista como algo atual e vem angariando força entre os atores do sistema, pois está intimamente ligada ao desenvolvimento do SUS na atualidade.

Dessa forma, a integração das ações educativas pautadas na EPS é de fundamental importância nas diferentes instâncias do SUS. Assim, a Atenção Primária à Saúde (APS), localizada próxima à população e responsável pela resolução da maioria dos problemas da população tem um papel estratégico na consolidação do sistema e da mesma maneira precisa estar preparada para essas ações. Tem, ainda, a possibilidade de utilizar os conceitos da EPS como ferramenta de trabalho, desde o planejamento até a avaliação de suas atividades.

Neste contexto, na realidade do Brasil, há a Estratégia Saúde da Família (ESF), a qual possui a responsabilidade de reorganizar a APS, se caracterizando como um dispositivo de expansão, qualificação e consolidação do SUS, além de beneficiar a reorientação do processo de trabalho, ampliando a resolutividade dos serviços de saúde, trabalhando com uma população adscrita, desenvolvendo ações de promoção à saúde, prevenção de doenças, diagnóstico e reabilitação de agravos ${ }^{3}$.

Nesta perspectiva, verifica-se facilidades e dificuldades na implementação das ações da ESF no cotidiano dos serviços de saúde. Muitas ações são viabilizadas com o suporte do processo de EPS o qual vem gerando importantes contribuições no contexto da APS e no trabalho em saúde. Dessa forma, se buscou pesquisar sobre esses diferentes processos que ocorrem junto aos trabalhadores e seus resultados no fazer em saúde.

Assim, este artigo é parte da dissertação de mestrado intitulada "Educação Permanente em Saúde e suas Implicações no Processo de Trabalho na Atenção Primária à Saúde" e tem o objetivo de identificar as contribuições da EPS no processo de trabalho da ESF de um município do sul do Brasil. O estudo foi realizado com as equipes de APS de um município do interior do RS, com os profissionais envolvidos no processo de EPS. Além do mais, segundo o Instituo Brasileiro de Geografia e Estatística ${ }^{4}$ mais de $73 \%$ dos municípios brasileiros possuem menos de vinte mil habitantes, perfil do município estudado, o que motiva a estudar realidades específicas e fomentar subsídios para a vivência da EPS nos demais municípios com o perfil semelhante. Este manuscrito tem como objetivo identificar as contribuições da educação permanente em saúde no processo de trabalho da estratégia saúde da família de um município do sul do Brasil.

\section{Método}

Trata-se do recorte de um estudo de mestrado, com abordagem qualitativa, que utilizou da técnica de pesquisa-ação para alcançar seus objetivos. Assim, a pesquisa qualitativa se ocupa de um nível de compreensão que não é possível de ser quantificado, ou seja, ela responde a questões particulares 5 . Já a pesquisa-ação é uma técnica de pesquisa não convencional que busca a intervenção, o desenvolvimento e a mudança, prevendo a interação entre o pesquisador e os atores envolvidos ${ }^{6}$.

Para facilitar a compreensão do processo de EPS na APS a pesquisa-ação auxiliou na busca de respostas e de alternativas com vistas a consolidação das práticas de EPS no processo de trabalho das equipes de saúde. Os participantes foram os profissionais 
de saúde lotados na Secretaria Municipal de Saúde (SMS) de um município do sul do Brasil, que participavam das ações de EPS, e que atuam na ESF ou nas equipes de apoio matricial, totalizando trinta e três profissionais de todos os níveis de formação (médio, técnico e superior). Sendo excluídos os profissionais que não pertenciam a equipes de ESF ou de apoio matricial ou que não participavam das ações de EPS. Para coleta de dados foi utilizado um questionário autoaplicável sobre o processo de EPS, como entendiam a educação permanente, sobre as experiências vivenciadas, quais contribuições o processo educativo gera no trabalho em saúde, as facilidades e as dificuldades notam neste processo, sendo realizada entre maio e outubro de 2016.

Em relação à assistência à saúde e a estrutura do SUS em nível municipal, havia na época da pesquisa, duas Unidades de Saúde da Família (USF); três equipes de saúde da ESF; duas equipes de apoio matricial, sendo uma equipe do Núcleo de Apoio à Atenção Básica (NAAB) e uma equipe do Núcleo de Apoio à Saúde da Família (NASF). Desde 2014, o município vivenciou um fortalecimento no processo de educação permanente junto às equipes, por meio da criação do Núcleo Municipal de Educação em Saúde Coletiva (NUMESC), o qual se tornou responsável pela coordenação, articulação e organização dos processos educativos em nível municipal.

Este artigo apresenta discussões a cerca das contribuições da EPS no processo de trabalho das equipes de saúde deste município. As informações advindas do questionário foram analisadas com base no processo de análise temática, sendo definida como o descobrimento dos núcleos de sentido que compõem uma comunicação, cuja presença signifique algo para o objeto de estudo ${ }^{7}$. As fases do processo de análise foram a pré-análise, a exploração do material, o tratamento dos resultados obtidos e a interpretação. Sendo assim, os dados foram sistematizados a partir dos questionários, gerando um quadro de análise com categorias. Neste texto são apresentados os resultados e discussões a cerca das contribuições da EPS no processo de trabalho junto aos usuários. A pesquisa foi aprovada pelo Comitê de Ética em Pesquisa da Universidade Federal de Ciências da Saúde de Porto Alegre (UFCSPA), mediante o parecer consubstanciado n. 1.459.159, de 21 de março de 2016. Assim, todos os participantes passaram pelo Processo de Consentimento Livre e Esclarecido de participação na pesquisa. Ainda, o financiamento foi próprio dos pesquisadores e não houve conflitos de interesses na execução deste estudo. Neste estudo, as contribuições apresentadas dizem respeitos a uma realidade específica, $o$ que pode ser considerado uma limitação importante, pois a partir dele não é possível realizar generalizações.

\section{Resultados e discussão}

Inicialmente, os trabalhadores da APS do município estudado afirmam que as ações de EPS colaboram fortalecimento do trabalho em saúde e no fortalecimento dos trabalhadores para atuar junto aos usuários. Como demonstrado a seguir:

Sim, contribui e muito, pois baseado nos casos estudados podemos usar na prática cotidiana com os usuários (Q1).

Me ajudou muito a ter mais confiança na relação com eles (Q2).

Nesses relatos os trabalhadores referem que, em suas práticas cotidianas, a EPS fortalece a relação com os usuários e o cultivo do vínculo com os mesmos. Os casos estudados durante esse processo estão associados à realidade vivenciada pelos profissionais e, devido a isso, as ações agregam um valor significativo para eles. O processo educativo apresenta um elemento transformador que auxilia os sujeitos a refletir sobre a sua própria realidade e os torna capaz de desenvolver e intervir no cotidiano ${ }^{8}$. 
Concomitantemente, a Portaria $\mathrm{n}^{\circ} 278$, de 27 de fevereiro de 2014, reafirma os princípios e as diretrizes da Educação Permanente como estratégia para a formação e o desenvolvimento dos trabalhadores, apostando no fortalecimento da EPS como ferramenta das práticas das equipes e dos contextos de trabalho, tendo como objeto de análise os processos de trabalho e como método a reflexão problematizadora, gerando processos de aprendizagem significativa'. Corroborando a isso, os trabalhadores associam o processo educativo a novas formas de fazer saúde. Assim, pode-se dizer que essa ferramenta provoca inquietações no trabalho cotidiano e nas interações, resultando em ações de saúde diferentes das vivenciadas nos cotidianos dos serviços de saúde, como ações educativas nas comunidades, atendimentos domiciliares, grupos terapêuticos, atividades coletivas, reuniões, entre outras.

EPS lança novos olhares sobre a forma de fazer o trabalho em saúde (Q4).

Compartilhamento das ações, é um trabalho vivo, é um trabalho em ato (Q3).

A respeito do trabalho vivo em ato, Merhy e Franco ${ }^{10}$ conceituam-no e auxiliam no processo de entendimento e experimentação de uma nova visão sobre o trabalho em saúde, que é essencialmente coletivo, pois depende da interação dos diferentes atores e tem como finalidade cuidar do outro. Esses novos olhares representam a superação do modelo biomédico na tentativa de alçar o olhar para a saúde da população, dos grupos e coletividades do território. Assim, a EPS colabora na superação dos mecanicismos vigentes, ampliando o conceito de trabalho em saúde e, consequentemente, qualificando os serviços.

Os trabalhadores apontam para a importância de o seu trabalho estar associado a essa finalidade: o cuidado do outro. Assim, o trabalho vivo proporciona inúmeras possibilidades de encontro com o usuário, pois tem a interação como a chave do cuidado e aponta para a construção participativa do projeto terapêutico, tudo isso baseado na singularidade do sujeito que está participando dessa troca, levando o trabalhador a aproximar suas atividades e a produção do cuidado às necessidades reais dos usuários. Podem-se observar dois recortes que apresentam essa percepção ${ }^{11}$.

Temos que compreender que o cuidado em saúde deve estar centrado no usuário (Q17).

[...] com certeza, pois não tem melhor que o usuário para realmente saber se o nosso objetivo é alcançado [...] (Q20).

A EPS, estando ligada ao conceito do trabalho vivo, colabora para o trabalho em saúde, pois é possível notar que não se trata de uma clínica centrada na patologia e na doença. Mesmo que essa superação ainda seja muito singela, é possível associar este fato ao investimento que este corpo de profissionais vem fazendo nos últimos anos em estratégias educativas com base na EPS.

Nesse interim, outras contribuições revelam aspectos relacionados à qualificação dos trabalhadores: questões de diálogo, entendimento e escuta.

Na maneira como conversar e entender (Q11).

Sim, pois um entendimento mais abrangente nos guia (Q24).

Com essas falas pode-se inferir que a EPS é fundamental para o trabalho em saúde, uma vez que, no atual cenário de busca na transformação do modelo assistencial é necessário desenvolver as tecnologias leves e as demais conforme cada projeto terapêutico. Tudo isso impacta na reestruturação produtiva da saúde, que é um modo diferente de produzir saúde, pois não está centrada no ato prescritivo, mas nos aspectos relacionais, afetivos e subjetivos dos sujeitos envolvidos ${ }^{11}$. 
Na perspectiva de aprofundar as relações e com isso qualificar o trabalho, os profissionais apresentam o que chamam de "trocas" como um fator contribuinte da EPS no fazer em saúde e em especial junto aos usuários, o que impacta nos serviços.

A troca de experiências, o compromisso com o público alvo fortalece os desafios encontrados, cria espaço de aprendizagem $(\mathrm{Q} 27)$.

[...], por permitir que com a troca de experiências entre todos os componentes possamos adaptar o serviço de acordo com a necessidade da população (Q29).

A partir disso, são criadas adaptações nos serviços de saúde tendo como guia as necessidades da população e, assim, os profissionais organizam-se para facilitar o acesso da população. Nesta atitude de troca é expresso o compromisso da equipe com os usuários e com o processo educativo, que se dá em todos os momentos, independentemente de ser um espaço dedicado para tal, ou seja, existem momentos de aprendizagem até mesmo na discussão das ofertas de serviços da equipe. Alguns autores apontam que as mudanças ocorrem quando os indivíduos têm a chance de revisitar suas ações e refletir sobre elas, buscando novas possibilidades ${ }^{12}$. Para tal, é necessário o envolvimento de todos, já que este momento de troca irá gerar um conhecimento novo e com significado para todos os sujeitos.

Reforçando essa questão um dos participantes da pesquisa refere que o saber da experiência vem sendo reforçado com a EPS.

[...] Se entendermos que a EPS éo saber da experiência, então significa que estamos falando e aprendendo junto do nosso usuário, pois nossas experiências estão diretamente ligadas ao usuário. Não tem como separar nossos aprendizados e conhecimentos do usuário (Q5).

Quando se traz o conceito do saber da experiência para a EPS, supera-se a lógica educativa tradicional e se depara com uma lógica que valoriza as individualidades, experiências e vivências dos sujeitos envolvidos no processo ${ }^{13}$. Tudo isso colabora para a construção de uma ação educativa libertadora que produz autonomia e satisfação, pois cria uma conexão intensa com a realidade vivida ${ }^{14}$, rompendo possíveis barreiras entre profissionais e usuários. Na mesma lógica, outro participante aponta que a EPS está associada ao trabalho, pois a mesma precisa surgir do trabalho e impactá-lo de certa forma.

\section{[...] A EPS sempre está associada ao nosso trabalho (Q23).}

Esse relato referenda o que é apresentado pela PNPES, uma política de formação e desenvolvimento para o SUS que valoriza a aprendizagem no trabalho, incorporando o aprender e ensinar ao cotidiano ${ }^{1}$, no sentido de transformar as práticas e qualificar os serviços como uma estratégia de superação dos problemas vivenciados pelas equipes.

Contribui com novas formas de ver o "problema", a situação vista por mais olhares sempre fica mais fácil de ser resolvida (Q26).

Contribui na superação de certos problemas detectados em equipe, podendo oferecer um melhor atendimento ao usuário (Q30).

Com base nesse relato e na bibliografia pode-se afirmar que existem lacunas na formação dos profissionais de saúde, como a falta de conhecimentos sobre o SUS e os processos educativos. A EPS auxilia na superação de diversos problemas e, ainda, é fundamental para o desenvolvimento e consolidação da ESF ${ }^{15}$. Dessa maneira, a EPS tem a característica de emanar da realidade vivenciada pelos profissionais e buscar suprir as necessidades na tentativa de qualificar os serviços ${ }^{16}$. Estes autores provocam a reflexão sobre a importância da EPS no tocante à resolução de problemas que fazem parte do cotidiano de trabalho dos profissionais da APS. 
Assim, um estudo evidencia que o dia a dia dos profissionais dá-se em um cenário de produção de problemas que são desafiados e resolvidos buscando a visão da EPS para agir e articular possíveis resoluções destes nós críticos corriqueiros ${ }^{17}$. Entretanto, a EPS é vista como algo complementar e não essencial no contexto do trabalho em saúde, como evidenciado neste recorte a seguir:

\section{É um complemento para o nosso trabalho (Q18).}

A partir desse recorte se entendem muitas das dificuldades encontradas na prática da EPS, pois não há um olhar para esse processo como algo essencial e necessário para o trabalho em saúde, que precisa caminhar conjuntamente ao fazer cotidiano. Poderia ser considerado complemento se não houvesse regulamentação em nível nacional, como política pública e estratégica de educação no trabalho, por meio da PNEPS ${ }^{1}$. Entretanto, existem dificuldades de compreensão por parte dos atores da real importância que a EPS possui no contexto do SUS. Outro estudo demonstra que as dificuldades de compreensão no que diz respeito à EPS caracterizam-se como um impasse para sua implementação ${ }^{18}$, ratificando o visualizado no fragmento citado.

Toda essa dificuldade se dá pelo fato de não compreender os princípios e diretrizes que regem essa política que permeia todo o fazer em saúde. Assim, pode-se transpor esse pensamento para a prática da APS. Ao realizar um acolhimento de um usuário na USF, está sendo trabalhando conceitos da EPS, ou seja, a forma como o que o usuário está relatando, como o profissional olha para o usuário, qual a é a postura do profissional diante do usuário, como se dá a construção do vínculo, o que será oferecido quando o usuário procura a unidade, qual a oferta que a unidade possui, qual é a demanda que o usuário apresenta, é possível a equipe trabalhar com este usuário para resolver o problema, entre outras questões. Tudo isso e muito mais é perceptível no contato do profissional com o usuário e com isso é possível notar que a EPS está presente nos mais variados espaços de trabalho em saúde, porém ainda é vista como complemento.

Nota-se, também, que alguns profissionais olham e vivem a EPS como uma transmissão de informações, lógica que busca ser superada a cada dia com novos conceitos de aprendizagem.

Conhecimento para eu levar aos usuários (Q12).

Me ajudando a transmitir aos usuários informações sobre os trabalhos que estão sendo oferecidos (Q28).

A partir disso é preciso refletir sobre alguns pontos importantes: que sentido tem esse conhecimento para o usuário? Ele é significativo? Ou simplesmente o profissional tem a obrigação de "transmitir"? É necessário encontrar o que faz sentido, o que dá sentido às informações, àquilo que será "inculturado", vivenciado pelo usuário e não apenas transmitir algo para cumprir uma obrigação legal. Aqui se verifica uma lógica educativa tradicional que não valoriza o sujeito envolvido e está baseada na teoria da transmissão de conhecimentos ${ }^{8}$.

Já em outro relato, nota-se que também se valoriza a experiência do usuário e que, com ela, os trabalhadores podem e aprendem muito.

Sim, porque tanto as ACS como profissionais tentam sempre levar informações para eles (usuários), mas também, ao mesmo tempo, ainda aprende muitas coisas com eles (Q19).

Essa percepção oportuniza a reflexão de que este aprendizado significativo faz efeito no fazer saúde na APS, pois as trocas se dão de forma instantânea. Assim como o profissional oferece a informação ele aprende com o usuário, e aí está uma riqueza que precisa ser explorada, que é a "conversa" entre os saberes acadêmico e popular. Dessa forma, a educação em serviço visa transformar as práticas pedagógicas, criando espaços de reflexão sobre a prática de trabalho, superando os limites da qualificação ${ }^{19}$. 
Entende-se que o fato de buscar manter uma postura dialógica com todos os atores, nos mais diversos espaços, abre caminhos para melhorias no campo da EPS na APS.

Entretanto, foi possível verificar que em alguns relatos há uma contradição, pois se notam alguns ruídos no que diz respeito à superação do modelo biomédico, sendo possível verificar algumas práticas deste saber nos discursos dos profissionais, em especial, quando falam em "levar" ou "transmitir" conhecimentos, demonstrando que o saber dos profissionais é superior ao dos usuários. Em relação às compreensões sobre as contribuições da EPS no processo de trabalho das equipes de APS há diversas, sendo em sua maioria contribuições propositivas e positivas para o trabalho em saúde e para a qualificação do mesmo.

\section{Conclusões}

Este artigo apresentou as principais contribuições da EPS no processo de trabalho da ESF, pela percepção dos trabalhadores de um município. Sendo assim, foi possível perceber que a EPS está associada ao fazer cotidiano destes trabalhadores, fortalecendo a equipe e sua relação entre colegas e com os usuários dos serviços de saúde. Ainda, auxiliando na construção de um cuidado centrado nos usuários, superando, de certa forma, o modelo biomédico hegemônico, uma vez que é possível notar uma postura de escuta desenvolvida pelos profissionais, facilitada pelas trocas no processo educativo.

Outro aspecto importante a ser destacado é a troca de experiências vivenciada pelos profissionais, com os seus pares e com os usuários, fato que colabora com a melhoria no acesso e na oferta de serviços, uma vez que estes aspectos são discutidos no processo de EPS. Entretanto, algumas dificuldades foram apontadas por alguns profissionais como: a visão da EPS como um complemento ao trabalho e não como algo intrínseco ao trabalho em saúde; e a compreensão do processo educativo em uma lógica tradicional, como a transmissão de conhecimento, de uma forma passival e vertical. Dificuldades que poderão ser superadas a partir de um maior investimento nas ações de educação no trabalho em saúde.

Nesse sentido, também foi apontado que a EPS favorece diferentes processos de valorização das experiências e vivências dos sujeitos envolvidos, uma vez que é baseado na aprendizagem significativa e na problematização, facilitando o acesso ao conhecimento, democratizando relações e saberes, a partir da prática educativa que é libertadora. Além do mais, promove o que se chama de empoderamento dos profissionais, pois todos têm possibilidades de expressão e liberdade para se colocar neste meio, o que facilita reflexões sobre o seu fazer cotidiano.

Por fim, demonstra o quão potente são os processos educativos no campo da saúde. E com isso, reforça a importância das experiências de EPS nos diversos cenários do SUS para que qualifique as ações e consolide o sistema como política pública. Reforçando a necessidade de investimentos no campo educacional em saúde para que o trabalho em saúde se torne cada vez mais democrático, acessível, de qualidade e que o ensinar-aprender-viver sejam indissociáveis.

\section{Referencias}

1. Brasil. Ministério da Saúde. Política Nacional de Educação Permanente em Saúde. Brasília (DF): Ministério da Saúde; 2009.

2. Pinto HA. Política nacional de educação permanente em saúde: aprender para transformar. In: Gomes LB, Barbosa MG, Ferla AA, organizadores. A educação permanente em saúde e as redes colaborativas: conexões para a produção de saberes e práticas. Porto Alegre (RS): Rede UNIDA; 2016. Capítulo 1.

3. Brasil. Ministério da Saúde. Política nacional de atenção básica. Brasília (DF): Ministério da Saúde; 2012.

4. Intituto Brasileiro de Geografia e Estatística [Internet]. Rio de Janeiro: IBGE Cidades; 2016 [citado em: 31 Ago 2016 ]. Disponível em: http://www.cidades.ibge.gov.br

5. Minayo MCS, Deslandes SF, Neto OC, Gomes R. Pesquisa Social: teoria, método e criatividade. Petrópolis (RJ): Vozes; 2011. 
6. Gil AC. Como elaborar projetos de pesquisa. São Paulo (SP): Atlas; 2010.

7. Minayo MCS. Desafio do conhecimento: pesquisa qualitativa em saúde. São Paulo (SP): Hucitec; 2014.

8. Freire P. Pedagogia do oprimido. Rio de Janeiro (RJ): Paz \& Terra; 2015.

9. Brasil. Ministério da Saúde. Portaria n. 278, de 27 de fevereiro de 2014. Institui Diretrizes para Implementação da Política de Educação Permanente em Saúde, no âmbito do Ministério da Saúde (MS). Diário Oficial da União, Brasília, DF, 28 fev. 2014. Seção 1.

10. Merhy EE, Franco TB. Trabalho em saúde. In: Escola Politécnica de Saúde Joaquim Venâncio organizador. Dicionário da educação profissional em saúde. Rio de Janeiro (RJ): EPJV; 2006. 308 p.

11. Franco TB, Merhy EE. Cartografias do trabalho e cuidado em saúde. Tempus. 2012;6(2):151-63. http://dx.doi.org/10.18569/ tempus.v6i2.1120

12. Barbosa VBA, Ferreira MLSM, Barbosa PMK. Educação permanente em saúde: uma estratégia para a formação dos agentes comunitários de saúde. Rev Gaúcha Enferm. 2012;33(1):56-63. http://dx.doi.org/10.1590/S1983-14472012000100008. PMid:22737796.

13. Educação Permanente em Saúde em Movimento [Internet]. Educação e trabalho em saúde: a importância do saber da experiência. [citado em: 27 nov. 2016]. Disponível em: http://eps.otics.org/material/entrada-textos/ educacao-e-trabalho-em-saude-a-importancia-do-saber-da-experiencia

14. Freire P. Pedagogia da autonomia. Rio de Janeiro (RJ): Paz \& Terra; 2015.

15. Paulino VCP, Souza PR, Borges CJ. Contribuições da educação permanente em serviço no contexto da estratégia de saúde da família. Itinerarius Reflectionis. 2010;2(9):1-11. https://doi.org/10.5216/rir.v2i9.1108

16. Silva LAA, Bonacina DM, Andrade A, Oliveira TC. Desafios na construção de um projeto de educação permanente em saúde. Rev Enferm UFSM. 2012;2(3):496-506. http://dx.doi.org/10.5902/217976925364.

17. Silva CT, Terra MG, Camponogara S, Kruse MHL, Roso CC, Xavier MS. Educação permanente em saúde a partir de profissionais de uma residência multidisciplinar: estudo de caso. Rev Gaúcha Enferm. 2014;35(3):49-54. http://dx.doi. org/10.1590/1983-1447.2014.03.44512. PMid:25474840.

18. Peres C, Silva RF, Della Barba PCS. Desafios e potencialidades do processo de educação permanente em saúde. Trab Educ Saúde. 2016;14(3):783-801. http://dx.doi.org/10.1590/1981-7746-sol00016.

19. Lopes SVLM, Freitas IR, Maciel MCC. Relato de Experiência: implantação do núcleo de educação permanente em Jataúba - PE. Rev Univ Vale Rio Verde. 2015;13(1):165-74. http://dx.doi.org/10.5892/ruvrd.v13i1.1941.

\section{Contribuição dos autores}

Guilherme Emanuel Weiss Pinheiro participou de todas as etapas da pesquisa e da confecção do manuscrito. Andrea Wander Bonamigo contribuiu na concepção da ideia, na coleta de dados, análise dos dados, acompanhamento, orientação do estudo e confecção do manuscrito. Marcelo Schenk de Azambuja contribuiu no acompanhamento, orientação do estudo e revisão do manuscrito. 